\title{
Aplicação da Análise Fatorial de Correspondências na Avaliação da Influência do Gesto na Performance Musical em Percussão[4]
}

\author{
Fernando Chaib \\ Dep. de Áreas Acadêmicas I - Instituto Federal de Educação, Ciência e Tecnologia de Goiás (IFG) \\ Rua 75, n.46, Centro te.74055-110. Goiânia (GO) \\ E-mail:fernandochaib@gmail.com
}

\author{
Homero Chaib Filho \\ EMBRAPA \\ Parque Estação Biológica - PqEB s/nº Brasília, DF - Brasil - CEP 70770-901 \\ E-mail: homerochaibfilho@gmail.com
}

\begin{abstract}
Resumo: Em uma performance percussiva, qual seria a influência dos gestos realizados pelo intérprete sobre as sensações de continuidade, suspensão e conclusão num expectador? Buscando responder essa questão, desenvolveu-se um experimento, denominado "Sensação de continuidade de um trecho musical". Este artigo apresenta como foi utilizada a Análise Fatorial de Correspondências (AFC) para corrrelacionar os "estímulos" com "os gestos" e "Fatores Contextuais", tendo por base os dados obtidos no experimento citado e que teve por finalidade observar aquela influência. Como resultado, percebe-se que em uma performance em percussão nos moldes apresentados no experimento, os gestos executados pelo instrumentista, durante a execução da obra, correlacionam-se às sensações dos espectadores.
\end{abstract}

1. Introdução. Considerando-se as sensações de continuidade (p.e.: quando existe uma ligadura de frase), suspensão (p.e.: quando existe uma fermata) e conclusão (p.e.: quando uma frase deve ser encerrada) contidas em uma obra para percussão, buscou-se evidenciar a influência dos gestos realizados pelo percussionista em uma performance musical sobre essas sensações. Para tanto notou-se a conveniência de se correlacionar os estímulos visuais, auditivos e audiovisuais com os gestos técnicos e expressivos e Fatores Contextuais (que expressam situações que podem 'desestabilizar' uma sensação) desenvolvidos para o experimento "Sensação de continuidade de um trecho musical" [5]. Os dados colhidos originalmente nesse experimento foram tratados e uma tabela foi montada para a realização da Análise Fatorial de Correspondências (AFC). Será possível perceber que ao ser realizada uma performance em percussão nos moldes apresentados no experimento em questão, os níveis de influência dos gestos executados durante a execução da obra poderão manter-se relacionados.

2. Um pouco sobre a AFC. Este trabalho trata-se de listar os tipos de respostas apresentadas no experimento realizado e, com a tabela de dados gerados, utilizar uma técnica de análise fatorial: "Dado que el problema de la medida en las ciencias sociales y del comportamiento adquiere especial dificultad, estos métodos, que relajan los requisitos exigibles a los datos, abren un amplio espectro de posibilidades para el estudio de estos fenómenos" [1]. Quando se emprega este tipo de análise busca-se observar o comportamento de uma variável ou grupos de variáveis em covariação com outras. As técnicas de análise multivariada são úteis para a descoberta de regularidades no comportamento de duas ou mais variáveis e para testar modelos alternativos de associação entre tais variáveis, incluindo a determinação de quando e como dois ou mais grupos diferem em seu perfil multivariado. No caso desse experimento, permite admitir que a relação estatística ali obtida poderá refletir-se para outros eventos envolvendo a performance para percussão, que não apenas os aqui reproduzidos.

A Análise Fatorial também é conhecida como Análise Multivariada. Tais técnicas visam ordenar e reduzir o número de variáveis correlacionadas entre si, através da geração de fatores, que associados aos auto-valores denominam-se componentes principais, permitindo a explicação da variabilidade do conjunto de dados iniciais [2]. A análise fatorial é essencialmente um método para determinar os fatores principais existentes em um conjunto de dados, definindo quais indivíduos ou variáveis pertencem a quais fatores, e com qual intensidade de pertinência tais indivíduos ou variáveis ocorrem em um fator. Existem duas 
vertentes essenciais para essas técnicas: uma que se baseia na estatística inferencial (abordagem anglo-saxônica); outra que se baseia na estatística descritiva (abordagem francesa). Os que se utilizam da abordagem francesa costumam chamar essas de técnicas de análise multidimensionais.

A AFC, uma das técnicas de análise multidimensional [6], é, por assim dizer, uma evolução da Análise de Componentes Principais (ACP) dirigida para a análise de tabelas em que os dados representam contagem. Na AFC, costuma-se representar a tabela de dados como uma matriz $n x m$, onde $n$ é o número de variáveis e $m$ o número de indivíduos. O conteúdo da matriz (ou tabela) é o número de ocorrências de um indivíduo em uma variável e a ela dá-se o nome de tabela de contingência. Desta forma a AFC gera fatores que agregam as variáveis ou indivíduos com seus respectivos graus de pertinência. Tais fatores têm uma hierarquia: $\mathrm{o}$, assim designado, primeiro fator é o que retém a maior quantidade de informações (ou o de maior peso das informações) contida na tabela de contingência; o segundo fator retém a segunda maior quantidade de informação; o terceiro, a terceira maior quantidade, e assim por diante. Esses fatores são, na verdade, eixos que definem espaços bidimensionais, tridimensionais, quadridimensionais ou $n$-dimensionais. Um plano fatorial é um espaço bidimensional formado por cada par de fatores gerados. Aquele composto pelos dois primeiros fatores chama-se primeiro plano fatorial e é o que agrega a maior parte das informações contidas na tabela de dados; é sobre o qual que, em geral, se atém a atenção da análise. Enquanto resultado, pode-se através dos fatores observar como as variáveis ou indivíduos relacionam-se dentro dos próprios fatores e no plano fatorial.

3. Sobre os dados - Experimento "Sensação de continuidade de um trecho musical"[5]. O experimento foi montado para três trechos subsequentes da obra Exil: Shangai 45, de Michel Longtin que, gravados, foram apresentados para três grupos de dois indivíduos cada, considerando-se os estímulos áudio (A), visual (V) e audiovisual (AV). Dois tipos de gestos foram utilizados para a gravação dos estímulos: Gesto Técnico (GT) e Gesto Expressivo (GE). Através de um PATCH desenvolvido com o software MAX/MSP 6.0, os participantes exprimiram suas sensações de continuidade, suspensão e conclusão, utilizando teclas coloridas no computador correspondentes a essas sensações. Cada grupo de indivíduos apreciou um estímulo, em cada trecho, combinado com um tipo de gesto (Tabela 1) [5]:

\begin{tabular}{|c|c|c|}
\hline In1 e In2 - GrI & In1 e In2 - GrII & In1 e In2 - GrIII \\
\hline A1t & A2t & A3t \\
\hline A $3 \mathrm{e}$ & A1e & A2e \\
\hline V2t & V3t & V1t \\
\hline V1e & V2e & V3e \\
\hline AV3t & AV1t & AV2t \\
\hline AV2e & AV3e & AV1e \\
\hline
\end{tabular}

Tabela 1: Divisão dos Estímulos por Indivíduso (In) e Grupos (Gr), onde: $\mathbf{A}, \mathbf{V}$ e $\mathbf{A V}$ são audio, visual e audiovisual; $\mathbf{1}, \mathbf{2}$ e $\mathbf{3}$ representa cada trecho e $\mathbf{t}$ (técnico), e (expressivo).

Efetuado o experimento, obtidas as respostas no programa e criadas as tabelas, como em Tabela1, definiu-se a letra $\boldsymbol{a}$ para designar a sensação de continuidade; a letra $\boldsymbol{b}$ sensação de suspensão e $\boldsymbol{d}$ para conclusão e combinados estímulos, gestos e trechos, foram obtidos 18 tipos de apresentações distintas gerando, ao todo, 36 respostas. Os dados obtidos do experimento receberam devido tratamento para permitir a montagem da tabela de contingência (Tabela 2) com vistas à realização da $\mathrm{AFC}$, onde:

- as linhas correspondem aos estímulos fornecidos no experimento realizado através das execuções técnica (t) ou expressiva (e): auditivo (At e Ae), visual ( $\mathbf{V t}$ e $\mathbf{V e}$ ) ou audiovisual (AVt e AVe).

- as colunas correspondem às sensações de continuidade (a), suspensão (b) e conclusão (d) em cada Fator Contextual (F).

Desta forma, cada célula da tabela expressa o número de respostas dadas à uma sensação dentro de um fator contextual devido a um estímulo. 


\begin{tabular}{|c|c|c|c|c|c|c|c|c|c|c|c|c|c|c|c|c|c|c|c|c|c|c|c|c|}
\hline & \multicolumn{3}{|c|}{ F1 } & \multicolumn{3}{|c|}{ F2 } & \multicolumn{3}{c|}{ F3 } & \multicolumn{3}{|c|}{ F4 } & \multicolumn{3}{|c|}{ F5 } & \multicolumn{3}{|c|}{ F6 } & \multicolumn{3}{|c|}{ F7 } & \multicolumn{3}{c|}{ F8 } \\
\hline Sensação & a & b & d & a & b & d & a & b & d & a & b & d & a & b & d & a & b & d & a & b & d & a & b & d \\
\hline Ae & 1 & 5 & 10 & 2 & 0 & 0 & 5 & 0 & 1 & 11 & 13 & 4 & 1 & 0 & 0 & 0 & 7 & 1 & 1 & 3 & 1 & 14 & 6 & 2 \\
\hline At & 3 & 8 & 4 & 2 & 0 & 0 & 4 & 5 & 2 & 6 & 11 & 4 & 0 & 0 & 0 & 4 & 5 & 0 & 0 & 1 & 3 & 20 & 9 & 2 \\
\hline Ve & 4 & 7 & 5 & 2 & 0 & 2 & 2 & 6 & 5 & 13 & 7 & 3 & 1 & 0 & 0 & 5 & 6 & 0 & 1 & 0 & 2 & 27 & 11 & 0 \\
\hline Vt & 3 & 7 & 7 & 2 & 2 & 0 & 4 & 7 & 0 & 9 & 8 & 0 & 0 & 1 & 0 & 2 & 9 & 1 & 0 & 0 & 1 & 16 & 16 & 2 \\
\hline AVe & 4 & 4 & 7 & 3 & 1 & 0 & 5 & 4 & 5 & 14 & 7 & 2 & 0 & 2 & 0 & 2 & 7 & 2 & 0 & 0 & 1 & 14 & 11 & 6 \\
\hline AVt & 2 & 4 & 9 & 1 & 1 & 0 & 7 & 3 & 1 & 10 & 16 & 6 & 1 & 0 & 0 & 3 & 5 & 1 & 1 & 2 & 1 & 19 & 3 & 2 \\
\hline
\end{tabular}

Tabela 2: Tabela de Contingência utilizada na AFC.

4. Utilização da AFC. Sobre os dados dessa tabela utilizou-se o software Statistical Analysis System (SAS) da Empresa Brasileira de Pesquisa Agropecuária (EMBRAPA). Considerando-se a definição da Tabela 2, suas colunas ao ser executado o software, foram nominadas como: F1a, F1b, F1d, F2a, F2b, e assim por diante até F8d. Desta forma, como exemplo, os elementos da coluna $\mathrm{F} 1 \mathrm{a}$ expressam o número de respostas dadas à sensação de continuidade (a) considerando o Fator Contextual F1 devido a um estímulo Ae, At,..., ou Avt. Semelhantemente, os elementos da coluna F4d expressarão o número de respostas dadas à sensação de conclusão (d) no Fator Contextual F4 para o estímulo Ae, At,..., ou Avt.

Considerando uma tabela de contingência, a AFC cria fatores que geram um espaço vetorial, no qual os pontos da tabela são projetados. Cada fator gerado retém uma quantidade de informação (associada ao peso relativo que os pontos possuem uns em relação aos outros na tabela de contingência) de maneira a que a soma da quantidade de informação de todos os fatores é igual a quantidade de informação total contida na tabela inicial.

Os fatores (eixos fatoriais) com maior quantidade de informação, são chamados "fatores principais", e são ordenados, decrescentemente, pela ordem de importância ou quantidade de informação retida pelo eixo fatorial. Os dois primeiros eixos fatoriais (os de maior peso), geram o primeiro plano fatorial que é o que retém maior quantidade de informação com respeito aos dados iniciais. Busca-se, então, um plano fatorial que retenha uma quantidade expressiva da informação total e considera-se que a partir de $70 \%$ da informação total, existe uma boa segurança para a realização da análise e estabelecimento das relações que extraem as informações dos dados da tabela de contingência. Ao se executar a AFC sobre a tabela equivalente à Tabela 2, o primeiro plano fatorial reteve aproximadamente $74 \%$ da informação total, sendo que o primeiro eixo reteve $42,73 \%$ e o segundo $30,74 \%$ (Figura 1 ).

Inertia and Chi-Square Decomposition

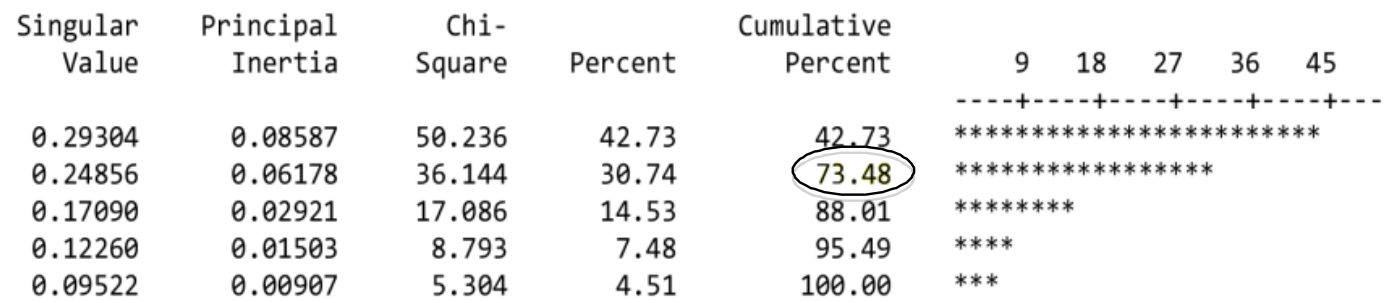

Figura 1: Decomposição da Inércia para a AFC.

O primeiro plano fatorial reteve informações suficientes para a caracterização dos eixos fatoriais e o estabelecimento de relações inter e entre as linhas (os estímulos) e colunas (as sensações para cada fator contextual). A seguir faz-se uma tipificação dos dois primeiros eixos em relação aos estímulos (linhas) e também em relação às sensações (colunas). Ressalta-se que o uso do termo "importância para a formação de um eixo" refere-se à caracterização com fins para a tipificação que permitirá a generalização dos resultados do experimento realizado.

4.1 Tipificação dos eixos fatoriais pelas linhas (estímulos). A Tabela 3 mostra a contribuição relativa de cada estímulo (quantidade de informação para a geração de um fator) 
para a formação dos dois primeiros eixos fatoriais a maneira como esses estímulos se correlacionam é evidenciada pelo sinal (+ ou -) de suas coordenadas no plano (Tabela 3):

\begin{tabular}{|l|c|}
\hline \multicolumn{2}{|c|}{ Contribuição Parcial } \\
\hline Estímulo & Eixo 1 \\
\hline Ae & $\mathbf{3 3 , 5 9}$ \\
\hline AVt & $\mathbf{3 1 , 1 8}$ \\
\hline Vt & $\mathbf{1 6 , 2 8}$ \\
\hline $\mathrm{Ve}$ & 9,52 \\
\hline $\mathrm{AVe}$ & 8,81 \\
\hline $\mathrm{At}$ & 0,62 \\
\hline
\end{tabular}

\begin{tabular}{|l|c|}
\hline \multicolumn{2}{|c|}{ Contribuição Parcial } \\
\hline Estímulo & Eixo 2 \\
\hline Ve & $\mathbf{3 7 , 1 2}$ \\
\hline AVe & $\mathbf{3 2 , 8 1}$ \\
\hline At & $\mathbf{1 6 , 3 0}$ \\
\hline Vt & 10,59 \\
\hline $\mathrm{Ae}$ & 3,05 \\
\hline $\mathrm{AVt}$ & 0,12 \\
\hline
\end{tabular}

\begin{tabular}{|l|c|c|}
\hline \multicolumn{3}{|c|}{ Sinal das Coordenadas } \\
\hline Estímulo & Eixo 1 & Eixo 2 \\
\hline At & - & - \\
\hline Vt & - & + \\
\hline AVt & + & - \\
\hline Ae & + & + \\
\hline Ve & - & - \\
\hline AVe & - & + \\
\hline
\end{tabular}

Tabela 3: Contribuição parcial das linhas (estímulos) para a formação do $1^{\circ} \mathrm{e} 2^{\circ}$ fatores

(eixos); sinais das coordenadas nos eixos fatoriais.

Vê-se que os estímulos que mais contribuíram para a formação do primeiro eixo (Eixo $1 \mathrm{em}$ Tab3 ) foram Ae, AVt e Vt, nesta ordem de importância; as coordenadas dos dois primeiros estímulos têm sinal positivo e a do terceiro negativo. Assim, com respeito ao primeiro fator, existe correlação positiva entre os estímulos Ae e AVt e esses dois estímulos têm correlação negativa com o estímulo $\mathbf{V t}$.

Vale lembrar que uma correlação positiva entre duas variáveis implica na mesma forma de variação entre elas: se uma aumenta a outra aumenta também; se diminui a outra diminuirá. Já a correlação negativa implica em variações contrárias: se uma aumenta a outra diminuirá e viceversa. Assim, pode-se dizer que o estímulo Ae provoca uma reação semelhante àquela provocada pelo estímulo AVt e que o estímulo $\mathbf{V t}$ provoca reação inversa aos dois anteriores. Isso significa que a magnitude das respostas dadas ao estímulo AVt é diretamente proporcional à magnitude das respostas dadas devido ao estímulo Ae. Por outro lado, as respostas dadas ao estímulo Vt têm magnitude inversamente proporcional às dadas aos estímulos Ae e AVt. Portanto, quando o indivíduo ouve e vê a execução técnica (AVt) faz uma associação equivalente àquela resultante da audição da execução expressiva (Ae). Porém, quando apenas vê, ele dá uma maior importância à execução técnica $(\mathbf{V t})$ sem relacionar a uma audição resultante de execução expressiva. Pode-se ver quais sensações relacionam-se predominantemente a estes estímulos pela associação das colunas aos eixos.

O segundo eixo fatorial (Eixo 2 em Tabela 3) está caracterizado pelos estímulos Ve (cuja coordenada é negativa), AVe (coordenada positiva) e At (coordenada negativa), nesta ordem de importância. Note-se que, para esse eixo fatorial, existe correlação positiva entre os estímulos Ve e At e negativa entre esses e o estímulo AVe. Tal fato indica que Ve e At provocam sensações semelhantes entre si e distintas de AVe.

Tais caracterizações permite tipificar o primeiro eixo fatorial como "do estímulo Ae e AVt". Os pontos correspondentes às colunas que tiverem coordenadas positivas no primeiro eixo corresponderão às sensações causadas preponderantemente por esses estímulos. O segundo eixo pode ser tipificado como "eixo associado ao GE (gesto expressivo)", sendo que os pontos correspondentes às sensações com sinal positivo no segundo eixo, serão causados preponderantemente pelo estímulo AVe; as sensações com sinal negativo nesse eixo, terão sido causadas preponderantemente pelo estímulo $\mathbf{V e}$. Ou seja, GE associa-se às sensações causadas pelos estímulos Ae e Ve, enquanto o estímulo $\mathbf{V e}$, corresponderá às sensações associadas à parte negativa do segundo eixo.

4.2. Tipificação dos eixos fatoriais pelas colunas (sensações). No que diz respeito à caracterização das colunas (sensações expressas devidos aos estímulos) constata-se que (Tabela 4), as sensações de maior peso na formação do primeiro eixo fatorial foram de suspensão (b)nos fatores contextuais F7, F8, F4, F3 e de conclusão (d)em F4, nesta ordem de importância para a formação desse fator. $\mathrm{O}$ sinal de $\mathrm{F} 7 \mathrm{~b}, \mathrm{~F} 4 \mathrm{~b}$ e $\mathrm{F} 4 \mathrm{~d}$, positivo, determina correlação positiva com Ae e AVt que, assim, são os principais estímulos a causar essas sensações. Para o aparecimento dessas sensações o estímulo $\mathbf{V t}$ teve muito pouca influência. O sinal negativo das coordenadas das sensações F8b e F3b nesse primeiro eixo, indica que praticamente não existirá influência dos estímulos Ae e AVt para o seu aparecimento, podendo sofrer leves influências do estímulo 
Vt. O segundo eixo fatorial foi gerado basicamente pelas sensações F8d, F2d, F8a, F5b, F6a e F6d, nessa ordem de importância. Desde que as sensações F8d, F5b e F6d têm suas coordenadas nesse segundo eixo positivas, estarão provocadas pelo estímulo AVe. As sensações F6a, F2d e F8a com coordenadas negativas no segundo eixo, foram provocadas pelo estímulo Ve.

\begin{tabular}{|l|l|}
\hline \multicolumn{2}{|c|}{ Contribuição Parcial } \\
\hline $\begin{array}{l}\text { Sensação por } \\
\text { fator (F) }\end{array}$ & Eixo 1 \\
\hline F7b & $\mathbf{1 6 , 3 9}$ \\
\hline F8b & $\mathbf{1 4 , 6 1}$ \\
\hline F4b & $\mathbf{1 4 , 5 2}$ \\
\hline F3b & $\mathbf{1 2 , 6 0}$ \\
\hline F4d & $\mathbf{1 0 , 1 5}$ \\
\hline F1d & 5,72 \\
\hline F3a & 3,95 \\
\hline F5b & 3,89 \\
\hline F3d & 3,11 \\
\hline F5a & 3,07 \\
\hline F7a & 3,07 \\
\hline F1a & 2,98 \\
\hline F2d & 1,76 \\
\hline F6a & 1,67 \\
\hline F2b & 0,87 \\
\hline F1b & 0,66 \\
\hline F2a & 0,35 \\
\hline F6b & 0,18 \\
\hline F7d & 0,16 \\
\hline F8d & 0,12 \\
\hline F6d & 0,08 \\
\hline F8a & 0,08 \\
\hline F4a & 0,02 \\
\hline
\end{tabular}

\begin{tabular}{|l|l|}
\hline \multicolumn{2}{|c|}{ Contribuição Parcial } \\
\hline $\begin{array}{l}\text { Sensação por } \\
\text { fator (F) }\end{array}$ & Eixo 2 \\
\hline F8d & $\mathbf{1 4 , 5 2}$ \\
\hline F2d & $\mathbf{1 3 , 5 7}$ \\
\hline F8a & $\mathbf{1 1 , 7 4}$ \\
\hline F5b & $\mathbf{1 1 , 6 6}$ \\
\hline F6a & $\mathbf{8 , 5 0}$ \\
\hline F6d & $\mathbf{8 , 5 0}$ \\
\hline F2b & 5,78 \\
\hline F1d & 4,07 \\
\hline F7d & 3,39 \\
\hline F4d & 2,62 \\
\hline F6b & 2,58 \\
\hline F3a & 2,42 \\
\hline F1b & 1,95 \\
\hline F2a & 1,85 \\
\hline F4a & 1,67 \\
\hline F8b & 1,64 \\
\hline F5a & 1,01 \\
\hline F7a & 1,01 \\
\hline F3b & 0,78 \\
\hline F3d & 0,66 \\
\hline F1a & 0,04 \\
\hline F4b & 0,01 \\
\hline F7b & 0,01 \\
\hline
\end{tabular}

\begin{tabular}{|l|c|c|}
\hline \multicolumn{3}{|c|}{ Coordenadas } \\
\hline $\begin{array}{c}\text { Sensação por } \\
\text { fator (F) }\end{array}$ & Eixo 1 & Eixo 2 \\
\hline F1a & - & - \\
\hline F1b & - & - \\
\hline F1d & + & + \\
\hline F2a & - & + \\
\hline F2b & - & + \\
\hline F2d & - & - \\
\hline F3a & + & + \\
\hline F3b & - & - \\
\hline F3d & - & - \\
\hline F4a & + & + \\
\hline F4b & + & - \\
\hline F4d & + & - \\
\hline F5a & + & - \\
\hline F5b & - & + \\
\hline F6a & - & - \\
\hline F6b & - & + \\
\hline F6d & + & + \\
\hline F7a & + & - \\
\hline F7b & + & + \\
\hline F7d & - & - \\
\hline F8a & - & - \\
\hline F8b & - & + \\
\hline F8d & - & + \\
\hline
\end{tabular}

Tabela 4: Contribuição parcial das sensações para a formação do $1^{\circ}$ e $2^{\circ}$ Eixos e sinais das coordenadas.

6. Interpretação do gráfico. Tendo sido feitas as tipificações dos dois primeiros eixos fatoriais, segundo estímulos e caracterizadas as sensações que têm maior importância para o surgimento desses eixos, pode ser feita a interpretação do gráfico do primeiro plano fatorial.

Antes, deve ser observado que os pontos no gráfico são projeções dos pontos originais definidos pela tabela de contingência. A AFC proporciona uma medida, chamada qualidade de representação do ponto no plano, definida pelo cosseno quadrado, que indica quão próximo ao plano está o ponto projetado. Essa medida varia de zero a um e diz que um ponto com qualidade de representação igual a 1, estará exatamente sobre o plano; quanto mais a qualidade de representação se aproxima de 0 , mais distante do plano estará o ponto. Tal informação é importante para que não haja confusão ao se estabelecer as relações entre pontos e eixos, na observação do gráfico desse plano. Quanto mais próxima de 1 for a qualidade de representação, mais firme será a relação entre o ponto e os eixos. A Tabela 5, traz, em ordem decrescente os valores para a qualidade de representação de linhas (estímulos) e colunas (sensações).

Vê-se que, à exceção de Vt e At, os demais estímulos estão muito bem representados, mostrando boa correlação entre os mesmos e o plano. Isso significa que os pontos das sensações que tiverem boa qualidade de representação terão a influência desses estímulos, como já indicado. Assim, considerando a qualidade de representação das sensações no plano, ao observarmos o gráfico, pode-se dizer qual estímulo provoca tal sensação e como esta sensação se correlaciona com as demais.

Como exemplo, tome-se a sensação F1d que tem qualidade de representação igual a 0,9. Pela observação do gráfico da AFC (Figura 2), nota-se que essa sensação está mais próxima do Eixo 2, pela parte positiva. Então, considerando a tipificação feita e a posição de F2a no gráfico 
(situa-se em cima do Eixo 2 pela parte positiva), pode-se dizer que a sensação de continuidade em F2 foi devido ao estímulo AVe.

\begin{tabular}{|c|c|c|c|c|c|}
\hline Estímulo & Qualidade & $\begin{array}{c}\text { Sensação por } \\
\text { Fator Contextual (F) }\end{array}$ & Qualidade & $\begin{array}{c}\text { Sensação por } \\
\text { Fator Contextual (F) }\end{array}$ & Qualidade \\
\hline Ae & $\mathbf{0 , 8 4}$ & F8a & $\mathbf{0 , 9 7}$ & F3a & $\mathbf{0 , 8 0}$ \\
\hline AVt & $\mathbf{0 , 8 4}$ & F2d & $\mathbf{0 , 9 3}$ & F8b & $\mathbf{0 , 7 9}$ \\
\hline Ve & $\mathbf{0 , 8 3}$ & F6d & $\mathbf{0 , 9 2}$ & F6a & $\mathbf{0 , 7 8}$ \\
\hline AVe & $\mathbf{0 , 7 1}$ & F5b & $\mathbf{0 , 9 2}$ & F8d & $\mathbf{0 , 6 6}$ \\
\hline Vt & $\mathbf{0 , 5 7}$ & F7b & $\mathbf{0 , 9 1}$ & F5a & $\mathbf{0 , 6 3}$ \\
\hline At & $\mathbf{0 , 5 4}$ & F1d & $\mathbf{0 , 9 0}$ & F7a & $\mathbf{0 , 6 3}$ \\
\hline & & F4b & $\mathbf{0 , 8 7}$ & F2a & $\mathbf{0 , 5 9}$ \\
\cline { 2 - 6 } & & F4d & $\mathbf{0 , 8 5}$ & F7d & $\mathbf{0 , 5 4}$ \\
\cline { 2 - 6 } & & F3b & $\mathbf{0 , 8 5}$ & F6b & $\mathbf{0 , 5 1}$ \\
\cline { 2 - 6 } & & F1a & $\mathbf{0 , 8 2}$ & F2b & $\mathbf{0 , 5 1}$ \\
\hline
\end{tabular}

Tabela 5: Qualidade de representação dos estímulos e das sensações no primeiro plano fatorial.

Os valores com qualidade de representação inferior a 50\% foram excluídos da tabela.

Outro exemplo poderá ser a leitura feita sobre F8a que tem a melhor qualidade de representação $(0,97)$, mas aparece pela parte negativa do Eixo 1. Ao considerar-se a mesma tipificação e posição de F6a no gráfico, percebe-se como esse fator surge próximo ao estímulo Ve, atestando sua influência sobre a sensação de continuidade em F6.

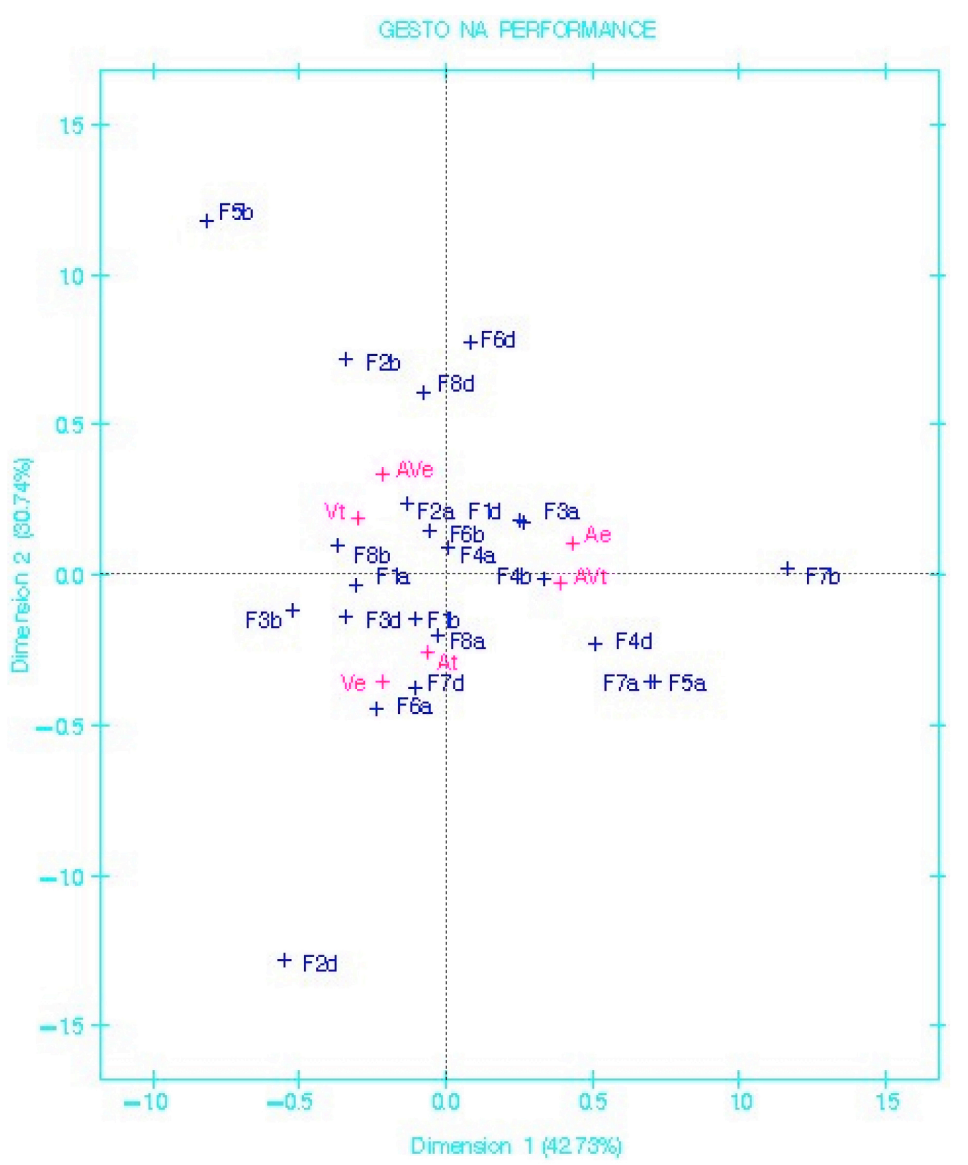

Figura 2: Gráfico da AFC, onde: Dimension $1=$ Eixo 1. Dimension $2=$ Eixo 3.

Pode-se dizer que as influências sofridas pelas sensações nos Fatores Contesxtuais (F) variaram de estímulo para estímulo, sendo que Ae e AVt tiveram mais peso nas sensações próximas ao Eixo 1 e Ve e AVe nas sensações próximas ao Eixo 2. Independentemente de ser GT ou GE, o estímulo audiovisual (AV) foi o que surgiu com maior peso nos dois eixos, em 
relação a $\mathbf{A}$ e $\mathbf{V}$, tendo maiores influências nas percepções das sensações de continuidade, suspensão e conclusão. Tratando-se propriamente de música, era de se esperar que o estímulo $\mathbf{A}$ se evidenciasse, porém é realmente relevante o resultado referente ao estímulo $\mathbf{V}$. Percebemos que, por esse prisma, o aspecto visual não pode ser ignorado na performance percussiva. É natural que, sobre esse ponto de vista e através dos elementos aqui apresentados, constate-se que todos os tipos de estímulos (A, V e AV) e dos gestos (GE e GT) presentes no experimento causaram, sob distintos níveis, influência nas sensações de continuidade, suspensão e conclusão a cerca dos trechos executados.

Nos resultados da AFC a expressividade da execução (GE), figurou com maior peso de influência representado pelos estímulos $\mathbf{A}, \mathbf{V}$ e $\mathbf{A V}$. Já o $\mathbf{G T}$ surge como agente influenciador através do estímulo AV (V e A tiveram pouco peso e menores níveis de influência aliados ao GT). Seguindo essa perspectiva e através daquilo que buscamos ilustrar relativamente às questões do GE e GT, percebe-se uma variação das influências em relação às três sensações do experimento. Como exemplo cita-se $\mathrm{F} 1$ que tem, na $\mathrm{AFC}$, a sensação de conclusão com maior influência a partir do estímulo AVe.

Os estímulos AVe e Ve condisseram com o sentido de continuidade nos fatores F2 e F6. O GE também surge como agente influenciador em F3, para a sensação de continuidade (sendo neste caso o estímulo V). Para F4 por exemplo, a sensação de suspensão obteve maior influência em AVt. A sensação de continuidade em F4 também teve maior peso através desse mesmo estímulo. Desta feita, pode se afirmado que, sendo GT ou GE, a visualização dos movimentos corporais (estando em convergência ou não com o estímulo auditivo) exerceram influência sobre as sensações de continuidade, suspensão e conclusão dos participantes do experimento sobre uma performance percussiva.

7. Impressões e conclusões sobre as análises realizadas. Do que se pôde analisar, será difícil afirmar que o GE, em conjunção com os estímulos, influenciará sempre a um nível preciso de sensação e o GT, da mesma forma, a um outro nível. Esses dois gestos parecem causar influências sobre as sensações de continuidade, suspensão e conclusão aliados também a contextos sonoros e musicais (timbre, meios expressivos, duração, etc.). Outrossim, a AFC assegura-nos que as relações das respostas poderão se manter, caso outros estudos sejam realizados sobre os mesmos parâmetros de performance, atestando a influência que o aspecto visual causa nas sesnações do espectador sobre a performance percussiva, estando em convergência com o estímulo auditivo (AV) ou de forma isolada (V).

\section{Referências}

[1] J.M. Batista, e J. Sureda, Análisis de correspondencias y técnicas de clasificación: Su interés para la investigación en las ciencias sociales y del comportamiento, Infancia y Aprendizaje n.39/40, pp.171-186 (1987).

[2] L. P. Barroso, Analise Multivariada, Colóquio na 48a Reunião da RBRAS e 10o SEGRO. Lavras. (2003).

[3] F. Chaib, Três Perspectivas Gestuais para uma Performance Percussiva: Técnica, Interpretativa e Expressiva, Per Musi n.27, pp. 159-181. Belo Horizonte (2013).

[4] "O Gesto na Performance em Percussão: Uma Abordagem Sensorial e Performativa". Tese de Doutorado. Departamento de Comunicação e Arte da Universidade de Aveiro. Aveiro (2012).

[5] F. Chaib, F. J. Catalão, H. Chaib Filho, A Influência do Gesto na Performance em Percussão: Análise Descritiva de Dados Experimentais, ANAIS do IX SIMCAM. Belém: ABCogMus (2013).

[6] L. Lebart; A. Moinea e M. Piron. "Statistique exploratoire multidimensionnelle". $3^{\mathrm{a}}$ Ed. Paris: Dunod (2002). 